OPEN ACCESS

Edited by:

Om V. Singh,

Technology Sciences Group Inc.

United States

Reviewed by:

Maria Guadalupe Vizoso Pinto,

Consejo Nacional de Investigaciones

Cientificas y Técnicas (CONICET),

Argentina

Jeanette Wagener,

University of Aberdeen,

United Kingdom

*Correspondence:

Zhennan Gu

zhennangu@jiangnan.edu.cn

Specialty section:

This article was submitted to

Food Microbiology,

a section of the journal

Frontiers in Microbiology

Received: 23 July 2018 Accepted: 04 February 2019 Published: 20 February 2019

Citation:

Liu J, Gu Z, Song F, Zhang H,

Zhao J and Chen W (2019)

Lactobacillus plantarum ZS2058

and Lactobacillus rhamnosus GG Use

Different Mechanisms to Prevent

Salmonella Infection in vivo.

Front. Microbiol. 10:299.

doi: 10.3389/fmicb.2019.00299

\section{Lactobacillus plantarum ZS2058 and Lactobacillus rhamnosus GG Use Different Mechanisms to Prevent Salmonella Infection in vivo}

\author{
Junsheng Liu'1,2,3, Zhennan Gu ${ }^{1,2 *}$, Fanfen Song ${ }^{1,2}$, Hao Zhang ${ }^{1,2,3}$, Jianxin Zhao ${ }^{1,2}$ and \\ Wei Chen $1,2,3,4$ \\ 'State Key Laboratory of Food Science and Technology, Jiangnan University, Wuxi, China, ${ }^{2}$ School of Food Science \\ and Technology, Jiangnan University, Wuxi, China, ${ }^{3}$ National Engineering Research Center for Functional Food, Jiangnan \\ University, Wuxi, China, ${ }^{4}$ Beijing Innovation Centre of Food Nutrition and Human Health, Beijing Technology and Business \\ University, Beijing, China
}

Pathogen-induced infectious diseases pose great threats to public health. Accordingly, many studies have investigated effective strategies targeting pathogenic infections. We previously reported the preventive effects of Lactobacillus plantarum ZS2058 (ZS2058) and L. rhamnosus GG (LGG) against Salmonella spp. in a murine model. Here, we compared the mechanisms underlying the preventive effects of these Lactobacillus strains in vivo. Notably, reduced C-reactive protein levels were observed with both ZS2058 and LGG, which suggests abrogated anti-infection and inflammatory responses. ZS2058 more efficiently reduced the pathogenicity of Salmonella by increasing the level of propionic acid in feces and production of mucin 2 in the mouse colon and activity through the interleukin (IL)-23/IL-22 and IL-23/IL-17 pathways. Meanwhile, LGG more strongly alleviated gut inflammation, as indicated by changes in the levels of tissue necrosis factor (TNF)- $\alpha, \mathrm{IL}-10$ and myeloperoxidase (MPO) in infected mice. Moreover, both ZS2058 and LGG restored the levels of interferon (INF)- $\gamma$, a cytokine suppressed by Salmonella, albeit through different pathways. Our results demonstrate that ZS2058 and LGG prevent Salmonella infection via different mechanisms.

Keywords: Lactobacillus, Salmonella, preventive effect, different, mechanism

\section{INTRODUCTION}

Salmonella infection, or salmonellosis, is associated with high morbidity and mortality and is therefore a significant public health concern worldwide. This problem is much more severe in developing countries because of the presence of contaminated food and water and poor sanitation facilities (Castillo et al., 2012). Therefore, investigations of effective strategies for coping with infectious diseases are highly significant.

Salmonellosis is usually treated clinically with antibiotics. However, these drugs can cause side effects, including antibiotic resistance and enteric dysbacteriosis. To date, some strains of multi-antibiotic-resistant Salmonella enterica serovar Typhimurium (S. Typhimurium) have been identified in poultry (Rajashekara et al., 2000). Other work has found that antibiotic therapy can 
exacerbate Salmonella-induced diarrhea and increase the period of pathogen shedding by at least 3 weeks (Neill et al., 1991). Accordingly, novel and safe strategies for salmonellosis prevention are vitally important.

Probiotics have been identified as a highly promising alternative treatment option for Salmonella infection because these products are associated with fewer side effects and better safety. Many studies have therefore investigated the ability of probiotics to prevent Salmonella infection, as well as the involved mechanisms. Several Lactobacillus strains were found to attenuate the intestinal epithelial barrier dysfunction induced by Salmonella lipopolysaccharide (LPS) (Yeung et al., 2013). L. rhamnosus S1K3 promotes the transcription of genes encoding Toll-like receptors in Peyer's patches (PPs) and modulates the levels of cytokines, which ultimately decreases the Salmonella load in mouse fecal matter and prevents bacterial invasion of internal organs (Kemgang et al., 2016). In a chicken model, L. salivarius CTC2197 was found to reduce Salmonella colonization (Pascual et al., 1999). Furthermore, the transcription of virulence genes identified as important contributors in Salmonella infection were reported to be modulated by Bifidobacterium thermophilum RBL67. This might indicate an important mechanism that could be targeted by probiotics to reduce pathogenicity and promote pathogen clearance (Tanner et al., 2016).

L. rhamnosus GG (LGG) is a well-established and widely recognized probiotic strain used extensively in scientific research and clinical applications. This strain exhibits strong antimicrobial activity against $S$. Typhimurium via the accumulation of lactic acid (De Keersmaecker et al., 2006). In a C3H/He/Oujco mouse model of infection, LGG reduces the population of $S$. Typhimurium, as well as the associated mortality (Hudault et al., 1997). Our previous studies demonstrated that both L. plantarum (L. plantarum) ZS2058 and LGG exhibited strong preventive effects against Salmonella-induced animal death in a mouse model (Liu et al., 2018). In this study, we investigated and compared the mechanisms by which ZS2058 and LGG prevent Salmonella infection. Notably, we found that ZS2058 and LGG each used several distinct pathways to prevent Salmonella infection.

\section{MATERIALS AND METHODS}

\section{Bacteria and Culture Conditions}

L. plantarum ZS2058 (ZS2058) and T (T), LGG and S. Typhimurium SL1344 (SL1344) were obtained from the Culture Collections of Food Microbiology (CCFM) at Jiangnan University (Wuxi, China). Lactobacilli and salmonellae were cultured, respectively, in MRS and $\mathrm{LB}$ broth $(0.3 \mathrm{M} \mathrm{NaCl})$ at $37^{\circ} \mathrm{C}$ unless otherwise stated.

\section{Animal Experiments}

Specific pathogen-free mice (SPF; C57BL/6, female, age: 68 weeks) were obtained from the Model Animal Research Center of Nanjing University and housed in a controlled room (SPF, constant temperature of $22^{\circ} \mathrm{C} \pm 2^{\circ} \mathrm{C}$ and humidity of $55 \% \pm 5 \%$ ) with a $12 \mathrm{~h}$ light-dark cycle at the Animal Experiment Center of Jiangnan University. This study was carried out in accordance with the recommendations of the European Community guidelines (Directive 2010/63/EU). The protocol was approved by the Ethics Committee of Jiangnan University.

Lactobacilli were washed with phosphate-buffered saline (PBS) and resuspended to a density of $5.0 \times 10^{9} \mathrm{CFU} / \mathrm{ml}$. Each mouse was administered $0.1 \mathrm{ml}$ of bacterial suspension or PBS (control and infection model groups) via gavage for 10 days. Subsequently, the mice were infected with $1.0 \times 10^{6} \mathrm{CFU}$ of $S$. Typhimurium SL1344 (Liu et al., 2018). At 2 day post-infection, the mice were sacrificed and samples of sera and intestinal tissue were collected and stored properly $(n=5$ or 6$)$.

To study the potential effects of lactobacilli on SCFAs production, in vivo experiments were designed to analyze SCFAs changes in feces of healthy mice. Mice received PBS (control group) or lactobacilli for 10 days $(n=5)$ without Salmonella infection. Feces were collected at different times (see Table 1) and the content of SCFAs were analyzed.

\section{C-Reactive Protein (CRP) Determination}

The serum levels of CRP were measured using enzyme-linked immunosorbent assay (ELISA) kits (Nanjing Senbeijia Biological Technology Co., Ltd., China).

\section{SCFAs Concentration in Feces}

Fecal concentrations of SCFAs were measured as previously reported ( $\mathrm{Li}$ et al., 2016). Briefly, feces were collected, weighed and freeze-dried. Subsequently, the fecal matter was soaked in saturated sodium chloride and treated with aqueous sulfuric acid and diethyl ether for acidification and extraction, respectively. The SCFA analysis was performed via gas chromatographymass spectrometry on a GCMS-QP2010 Ultra device (Shimadzu Co., Tokyo, Japan).

\begin{tabular}{lcccc}
\hline \multicolumn{4}{l}{ TABLE 1 | } & Analysis of short chain fatty acids (SCFAs) in mice feces ${ }^{\mathrm{a}}$. \\
\hline $\begin{array}{l}\text { SCFAs, } \\
\boldsymbol{\mu} \mathbf{\text { mol/g}}\end{array}$ & Group & $\begin{array}{c}\mathbf{1 0} \text { days of } \\
\text { gavage }\end{array}$ & $\begin{array}{c}\mathbf{7} \text { days } \\
\text { post-gavage }\end{array}$ & $\begin{array}{c}\mathbf{1 4} \text { days } \\
\text { post-gavage }\end{array}$ \\
\hline Acetic acid & Control & $77.01 \pm 3.58$ & $73.70 \pm 5.72$ & $65.53 \pm 5.51$ \\
& ZS2058 & $92.72 \pm 9.89$ & $86.37 \pm 6.08$ & $70.29 \pm 4.48$ \\
& $\mathrm{~T}$ & $97.33 \pm 4.85$ & $73.99 \pm 4.92$ & $79.54 \pm 11.71$ \\
& LGG & $81.78 \pm 8.90$ & $68.52 \pm 3.83$ & $80.55 \pm 12.24$ \\
Propionic & Control & $10.66 \pm 0.54$ & $11.59 \pm 0.77$ & $11.76 \pm 1.19$ \\
acid & & & & \\
& ZS2058 & $18.63 \pm 2.00^{*}$ & $15.32 \pm 5.42$ & $10.22 \pm 0.91$ \\
& $T$ & $12.91 \pm 3.74$ & $12.59 \pm 3.64$ & $10.01 \pm 0.76$ \\
Butyric acid & LGG & $13.40 \pm 4.06$ & $10.05 \pm 1.34$ & $12.68 \pm 0.78$ \\
& ZS2058 & $8.31 \pm 2.11$ & $9.60 \pm 1.58$ & $7.65 \pm 2.02$ \\
& $T$ & $6.24 \pm 2.06$ & $7.69 \pm 2.77$ & $6.99 \pm 2.77$ \\
& LGG & $7.77 \pm 1.98$ & $5.70 \pm 1.55$ & $5.82 \pm 2.34$ \\
\hline
\end{tabular}

${ }^{a}$ Data were analyzed by using T-test, $n=5 .{ }^{*} p<0.05$ vs. Control. 


\section{Determination of the Levels of Mucin 2 (MUC2), Myeloperoxidase (MPO), and Cytokines}

The stored samples of intestines were homogenized at a 1:9 (m/v) dilution in cold PBS. The levels of MUC2, MPO and various cytokines [tissue necrosis factor (TNF)- $\alpha$, interleukin (IL)-23, IL22, IL-17, IL-10, interferon (IFN)- $\gamma$, IL-18, IL-2, IL-1 $\alpha$, IL-12, and transforming growth factor (TGF)- $\beta$ ] in these homogenates and the stored serum samples were then determined using ELISA kits (Nanjing Senbeijia Biological Technology Co., Ltd.).

\section{Body, Spleen and Liver Weights, and Organ Relative Ratios}

Body weights (BWs) and organ weights were obtained at different time points or at dissection. The organ relative ratio was calculated as the g/g BW (Yoshimi et al., 2001).

\section{Statistics}

Data are expressed as means \pm standard errors of the means. Mean values of different groups were analyzed using a oneway variance analysis (one-way ANOVA) with Duncan's multiple range tests with SPSS 16.0 Statistical Software (IBM Corporation, Armonk, NY, United States). Data were considered to be statistically different at a $P<0.05$, and were indicated by different superscript letters (such as a, b, and c). Differences between means that do not share a letter are statistically significant.

\section{RESULTS}

\section{Both ZS2058 and LGG Reduced CRP Levels in Infected Mice}

An infection is an important source of inflammatory stimuli and thus promotes the production of CRP (McDade et al., 2008), which can then be used clinically as a diagnostic parameter for infection and bacterial sepsis. As shown in Figure 1, Salmonella-infected mice exhibited significantly increased CRP levels (179.1 vs. $163.2 \mu \mathrm{g} / \mathrm{L}$ in the uninfected group). Both ZS2058 $(161.4 \mu \mathrm{g} / \mathrm{L})$ and LGG treatments $(161.8 \mu \mathrm{g} / \mathrm{L})$ were associated with reduced levels of CRP, indicating an alleviation of Salmonella infection in vivo. Our results demonstrate that pretreatment with either Lactobacillus strain could reduce CRP levels in the sera of infected mice.

\section{ZS2058 Showed More Efficiency in Reducing the Pathogenicity of Salmonella in Intestinal Phase}

\section{ZS2058 Increased Propionic Acid Level in Feces}

The increased research interest in probiotics and prebiotics has directed increasing levels of attention toward SCFAs. As shown in Table 1, PA levels in fecal matter were significantly increased after a 10 days course of ZS2058 gavage $(18.63 \mu \mathrm{mol} / \mathrm{g})$ when compared with the PBS-treated (control) group $(10.66 \mu \mathrm{mol} / \mathrm{g}$ ). Other treatments did not induce any significant changes in the SCFA profile at any time post-gavage (Table 1). At 7 and 14

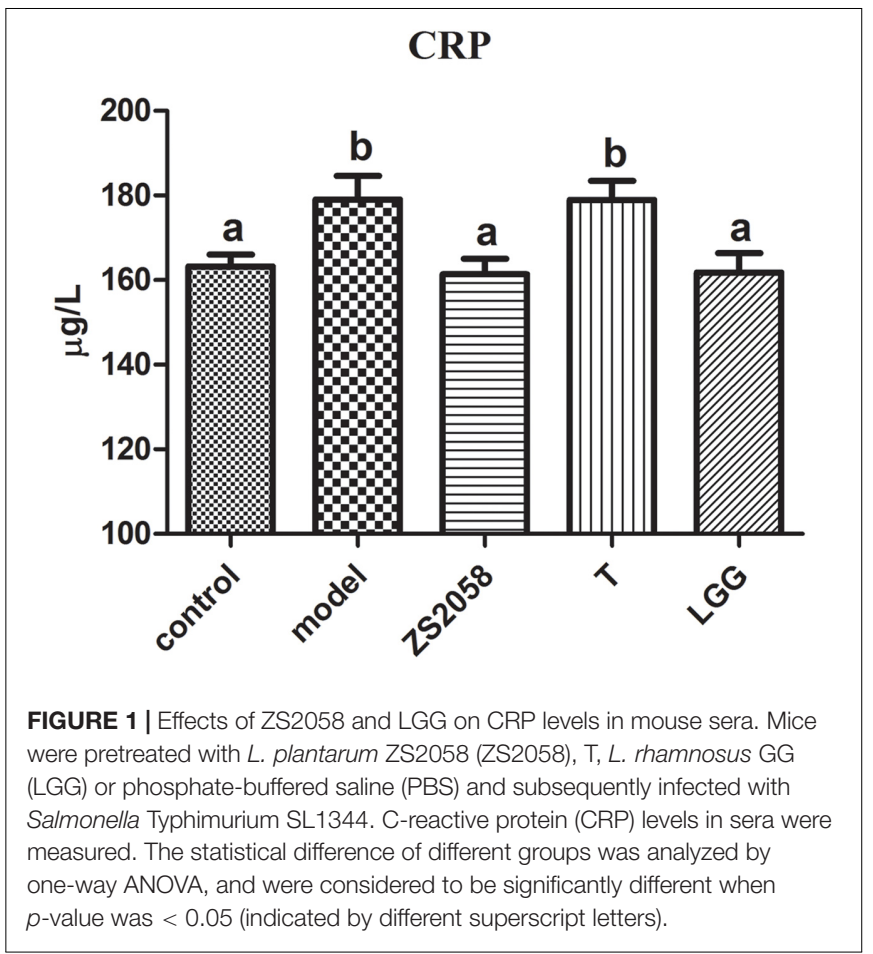

days post-gavage, no changes in the tested SCFAs were observed after treatment with ZS2058, T or LGG. Our results therefore demonstrate that ZS2058 more strongly affected PA production. As this SCFA has been reported to limit Salmonella colonization in vivo (Alshawabkeh and Tabbaa, 2002), our finding suggests that ZS2058 might protect the host from infection by increasing the production of PA.

\section{ZS2058 Reduced the Level of MUC2 in Infected Mice} A mucus layer covers the intestinal surfaces to form a physiological barrier that excludes luminal bacteria. Several mucins form the gel-forming glycoprotein component of this barrier, of which MUC2 has been identified as the major contributor to the colonic mucus layer (Kumar et al., 2017). As shown in Figure 2, infection with S. Typhimurium SL1344 significantly increased the colonic MUC2 levels. However, pretreatment with LGG reduced the production of MUC2 $(2.06 \mathrm{ng} / \mathrm{g})$ to a level comparable with that in the uninfected group (2.06 ng/g). However, ZS2058 pretreatment significantly reduced the MUC2 level relative to the uninfected and model groups (1.93 ng/g) (Figure 2). In summary, ZS2058 more effectively reduced MUC2 production in Salmonellainfected mice.

\section{ZS2058 More Strongly Promoted Activity Through the IL-23/IL-22 and IL-23/IL-17 Axes}

IL-23 is a critical cytokine associated with host innate immune responses against Salmonella. This cytokine can induce IL17 and IL-22, which are involved in the rapid response to infectious agents (Valeri and Raffatellu, 2016). In the ileum, IL-23 levels decreased significantly in response to infection (0.528 vs. $0.594 \mathrm{ng} / \mathrm{g}$ in the uninfected group; Figure 3A), whereas ZS2058 


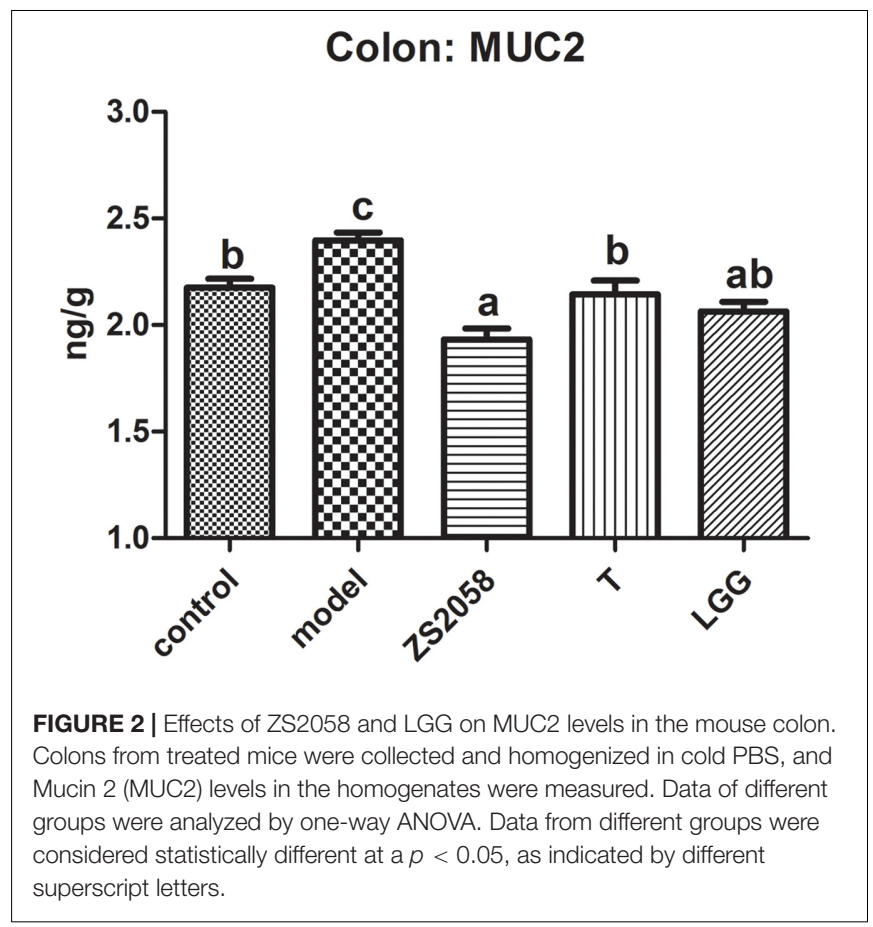

pretreatment restored IL-23 levels in this organ $(0.607 \mathrm{ng} / \mathrm{g}$, Figure 3A). By contrast, SL1344 infection had no significant effects on the levels of IL-22 (Figure 3B) and IL-17 (Figure 3C). Both ZS2058 and T (Figure 3B) significantly promoted IL-22 production, whereas IL-17 expression was significantly decreased by $\mathrm{T}$ (Figure 3C) and slightly decreased by LGG (Figure 3C). These results demonstrate the ability of ZS2058 to promote the IL23/IL-22 axis in the mouse ileum.
In the colon, both Salmonella infection and ZS2058 pretreatment significantly increased the levels of IL-23, whereas LGG had no significant effect (Figure 3D). Regarding IL-22, the ZS2058- and LGG-treated mice, respectively, exhibited a slight increase and slight decrease (non-significant) in the levels of this cytokine (Figure 3E) when compared with PBS-treated and infected mice. Regarding IL-17, S. Typhimurium SL1344 infection significantly reduced the expression of this cytokine in the colon (Figure 3F). However, ZS2058 significantly increased the colonic level of IL-17 relative to the control group, whereas LGG only restored this cytokine to a comparable level with the control (Figure 3F). Our results demonstrate that ZS2058 more strongly promotes the IL-23/IL-17 axis in the mouse colon.

\section{LGG More Effectively Alleviated Gut Inflammation}

Pathogenic infections usually cause gut inflammation, which can be detected by changes in the cytokine profile. As shown in Figure 4A, the levels of the pro-inflammatory cytokine TNF- $\alpha$ in the mice colon increased significantly in response to infection, but were significantly reduced by pretreatment with both ZS2058 and LGG. The levels of IL-10, an important anti-inflammatory cytokine, were slightly increased by infection (Figure 4B) and significantly reduced by ZS2058, but maintained at a comparable level with that in infected mice by LGG (Figure 4B). The level of $\mathrm{MPO}$, a marker of neutrophil infiltration during inflammation, was not significantly affected by infection or pretreatment with ZS2058 or T, but was significantly reduced by LGG (Figure 4C). In summary, only LGG reduced the levels of TNF- $\alpha$ in the colon while maintaining IL-10 and significantly reducing MPO (Figure 4C). These results suggest that LGG more effectively alleviates gut inflammation.

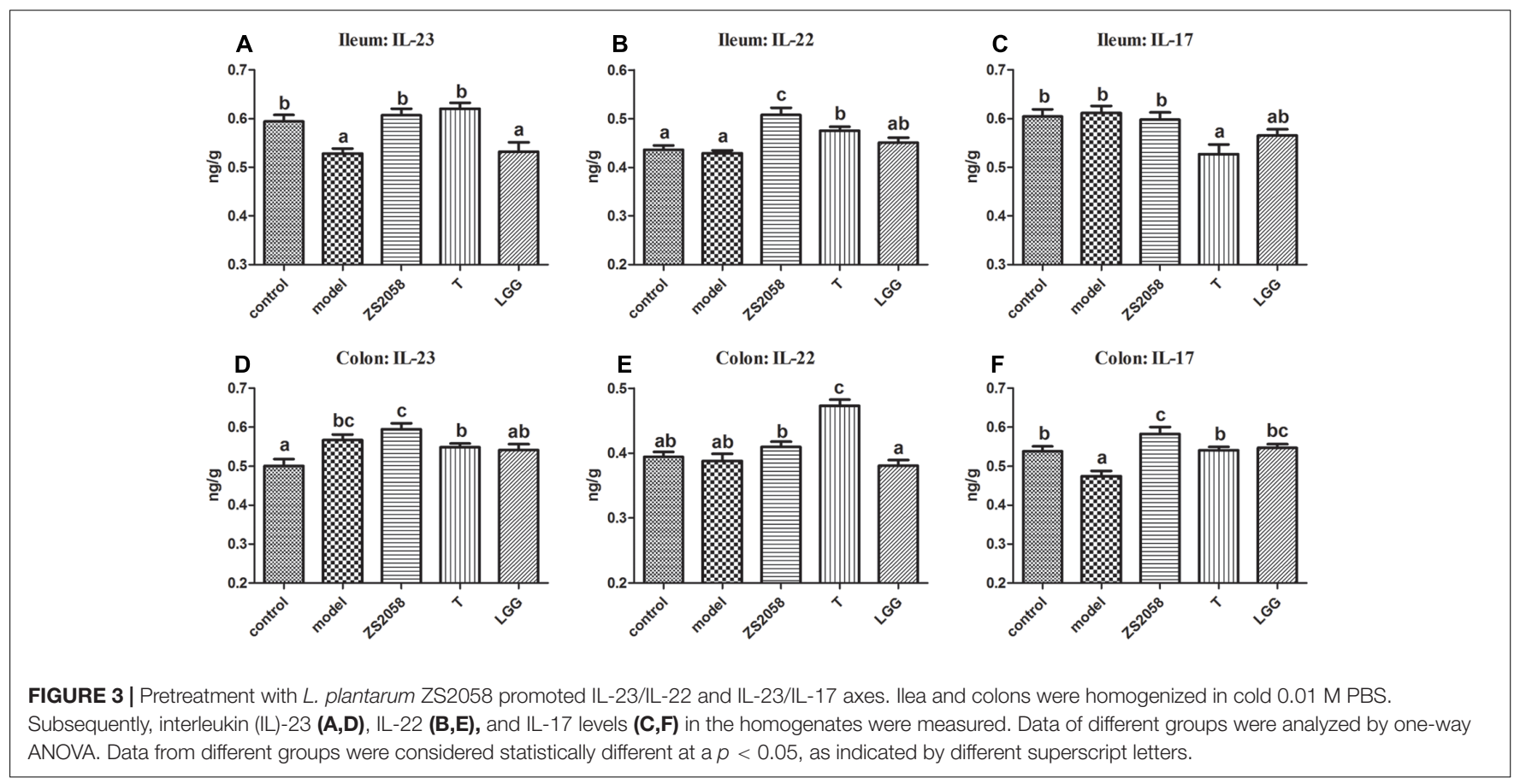



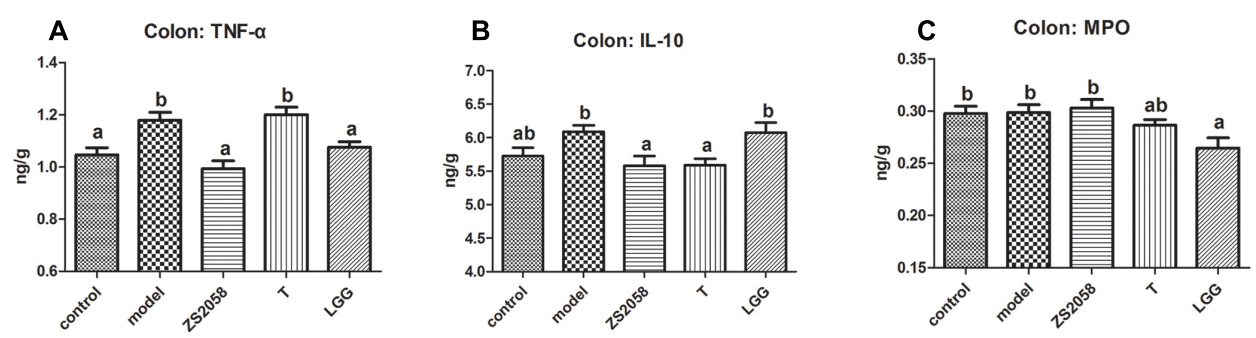

FIGURE 4 | Effects of L. plantarum ZS2058 and L. rhamnosus GG (LGG) on gut inflammation. Colons were collected from treated mice and homogenized with cold PBS. Tumor necrosis factor (TNF)- $\alpha$ (A), interleukin (IL)-10 (B), and myeloperoxidase (MPO) (C) levels were measured in the homogenates. Means of different groups were considered statistically different at a $p<0.05$ by analysis of one-way ANOVA, and indicated by different superscript letters.

\section{ZS2058 and LGG Restored IFN- $\gamma$ Through Different Pathways}

IFN- $\gamma$, a characteristic cytokine in a Th1-type response, may play a major role in enhancing the anti-bacterial activities of macrophages. An in vivo study found that IFN- $\gamma$ deficiency led to an increased splenic Salmonella load and decreased survival rate in mice, while treatment with IFN- $\gamma$ prevented the deterioration associated with infection (Eckmann and Kagnoff, 2001). As shown in Figure 5A, the uninfected mice in the control group had a serum IFN- $\gamma$ level of $1319.4 \mathrm{ng} / \mathrm{L}$. This level decreased significantly to $1114.9 \mathrm{ng} / \mathrm{L}$ in response to $S$. Typhimurium SL1344 infection. However, pretreatment with ZS2058 (1250.4 ng/L) or LGG (1271.0 ng/L) restored the IFN- $\gamma$ levels to comparable with those in the control group (Figure 5A). We subsequently evaluated the levels of IL-12 and IL-18, which induce the production of IFN- $\gamma$. Notably, ZS2058 significantly increased the production of IL-18 (Figure 5B) while LGG significantly increased the production of IL-12 (Figure 5C), indicating that these probiotic strains restored IFN- $\gamma$ levels through distinct pathways.

\section{DISCUSSION}

Globally, salmonellosis is associated with high rates of morbidity, hospitalization, and mortality. A loss of body weight, splenomegaly and hepatomegaly are all typical symptoms of salmonellosis. In this study, we found that infected mice did not exhibit any of these symptoms (Figure 6) during the early stage of infection (2 days post-infection), indicating that $S$. Typhimurium SL1344 did not cause severe systemic infection at this time point.

As noted above, CRP is a useful diagnostic marker of infection. In effect, CRP can be produced in many types of inflammation (Ridker, 2003). We observed that the serum CRP level increased significantly in response to S. Typhimurium SL1344 infection but decreased in mice that were pretreated with ZS2058 or LGG. We previously reported that L. plantarum ZS2058 and L. rhamnosus GG, but not L. plantarum T, could significantly reduce Salmonella-related deaths in a murine model. Our results provide further evidence of the preventive effects of ZS2058 and LGG against Salmonella infection. The reduced levels of CRP in infected mice treated with both probiotic strain suggests that the inflammatory responses are alleviated systemically. Although LGG appeared to more strongly alleviate gut inflammation, ZS2058 appeared to be a more effective regulator of systemic inflammation resulting from Salmonella.

Following oral infection, pathogens pass through the gastrointestinal tract, where they colonize, survive, replicate and initiate the invasion process. In the gut lumen, the host microbiota plays a crucial role in the inhibition of invasive pathogens through processes including the fermentation of certain carbohydrates to produce SCFAs. In an analysis of the fecal SCFA contents, we found that after a 10 -d treatment, only
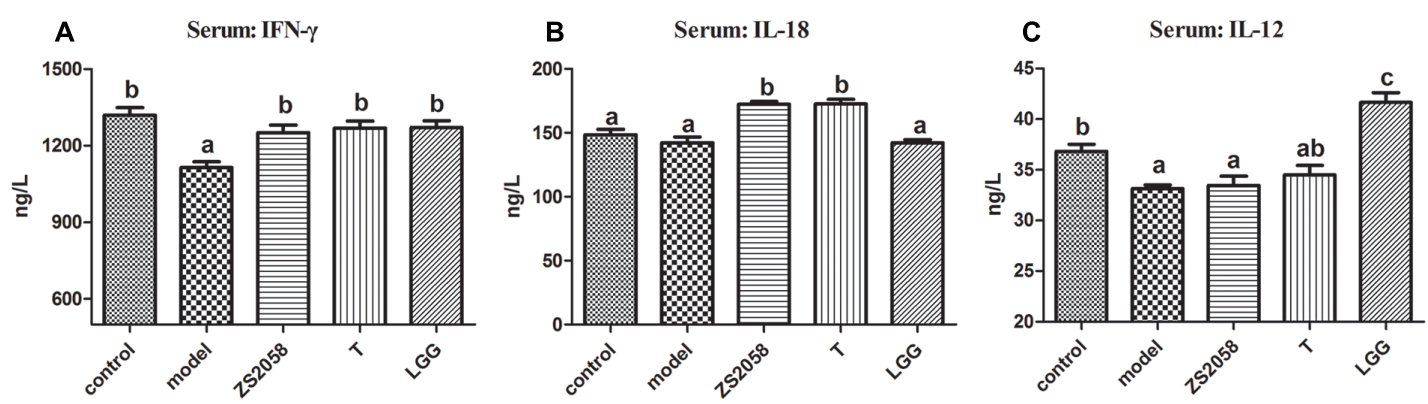

FIGURE 5 | L. plantarum ZS2058 and L. rhamnosus GG (LGG) restored interferon (IFN)- $\gamma$ production via different pathways. Interferon (IFN)- $\gamma$ (A), interleukin (IL)-18 (B), and IL-12 (C) levels were measured in sera collected from mice at the time of sacrifice. Means of different groups were considered statistically different at a $p<0.05$ by analysis of one-way ANOVA, and indicated by different superscript letters. 

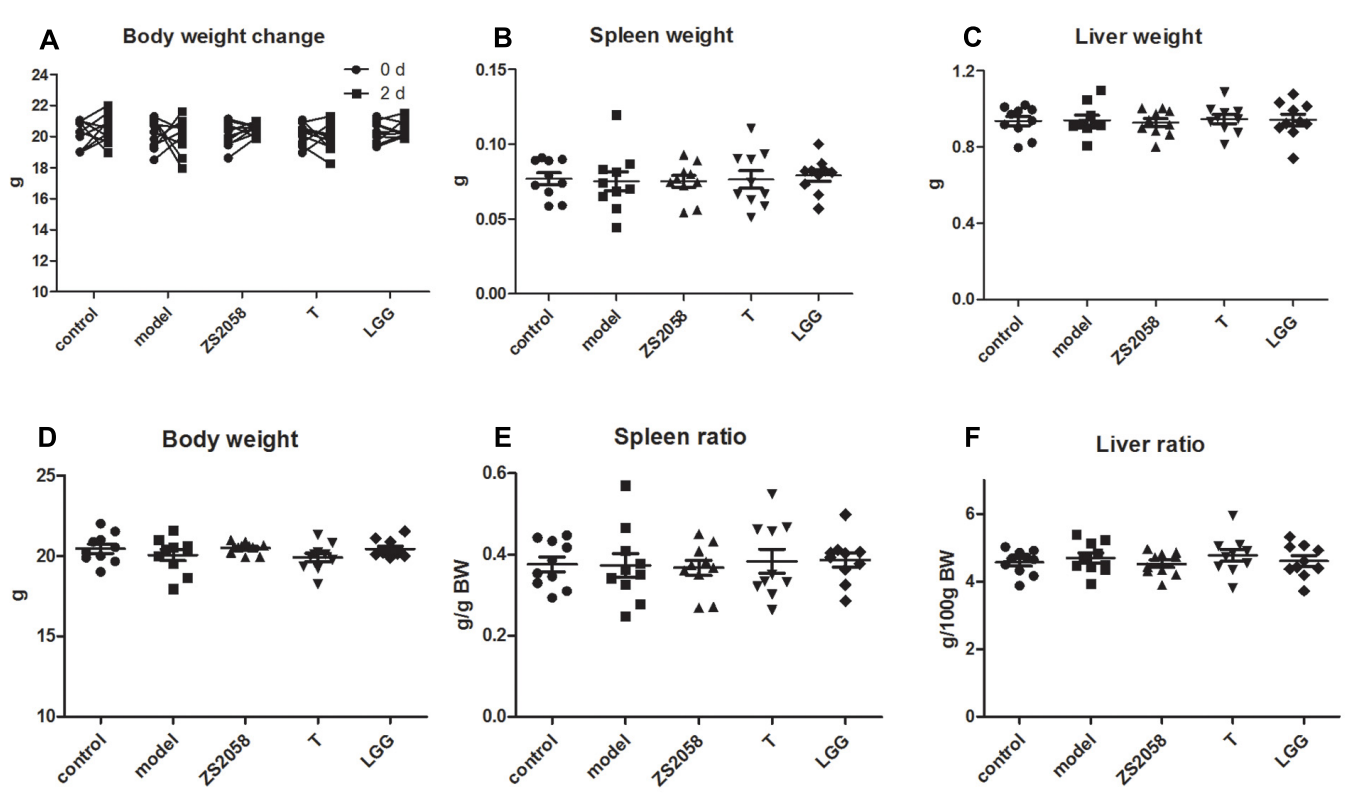

FIGURE 6 | Mice did not exhibit typical symptoms of salmonellosis during the early stage of infection. Mice body weight (A-D) were measured immediately after infection and at 2 days post-infection. Spleen (B), and liver (C) weight were measured at sacrifice; spleen ratio (E), and liver ratio (F) were calculated.

ZS2058 led to a significant increase in the PA level (Table 1). However, ZS2058, T and LGG all tended to increase the levels of acetic acid, PA and butyric acid (Table 1). PA is reported to alter the expression of $S$. Typhimurium genes associated with invasiveness (Lawhon et al., 2002), and to reduce Salmonella colonization in the gastrointestinal tract (Levison, 1973). In vivo studies have shown that SCFAs promote host defense against Salmonella and reduce pathogen loads in the intestinal contents
(Sunkara et al., 2011). Accordingly, the ability of ZS2058 to promote the production of PA might contribute to the preventive effects of this probiotic in vivo. Increased levels of SCFAs, such as PA, might comprise an important mechanism by which probiotics mediate anti-Salmonella functions. Although treatment with lactobacilli might alter the profile or increase the abundance of certain species of host microbiota, a limited substrate might not allow excessive changes in SCFAs. Additional
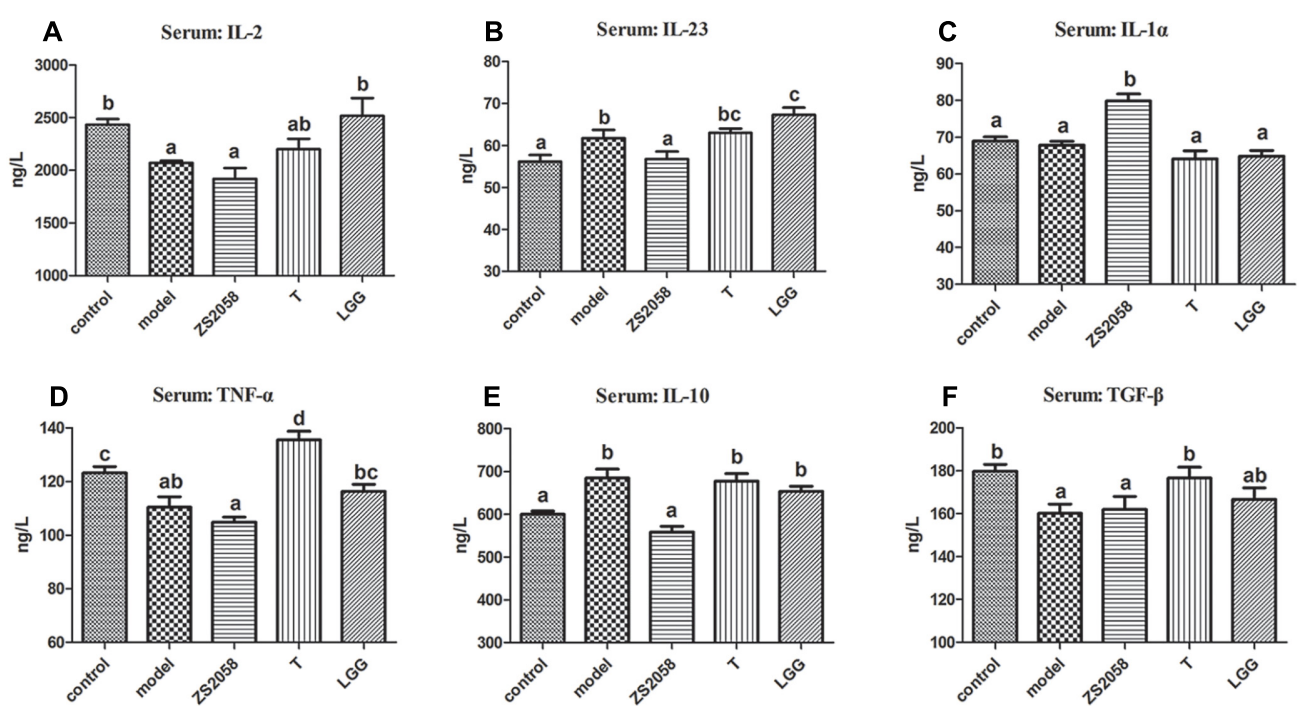

FIGURE 7 | Different effects of $L$. plantarum ZS2058 and L. rhamnosuS GG (LGG) on the cytokine profiles in sera from infected mice. Sera were collected from mice sacrificed at 2 days post-infection and subjected to ELISAs to detect IL-2 (A), IL-23 (B), IL-1 $\alpha$ (C), TNF- $\alpha$ (D), IL-10 (E), and TGF- $\beta$ (F). Means of different groups were considered statistically different at a $p<0.05$ by analysis of one-way ANOVA, and indicated by different superscript letters. 
supplementation with substrates, especially prebiotics which are mostly fermented in the colon, might be an effective way to promote the production of SCFAs.

Besides the above-listed effects, SCFAs also contribute to gut immune homeostasis. For example, butyrate can modify the production of II-12 and IL-23 (Berndt et al., 2012). IL-23 induces the production of IL-22 and IL-17 in several cell types, including Th17 cells and NKT cells. In turn, IL-22 can induce the expression of iNOS, the siderophore lipocalin-2 and MUC4 (Raffatellu et al., 2009), while IL-17 recruits neutrophils that play a crucial role in host defense against extracellular bacteria (Santos et al., 2009). We found that in the ileum, LGG treatment did not significantly affect the IL-23/IL-22 (Figures 3A,B) and IL-23/IL17 (Figures 3A,C) axes when compared with the Salmonellainfected group, whereas ZS2058 had a stronger positive effect on the IL-23/IL-22 (Figures 3A,B) axis when compared with T. LGG-treated mice exhibited slight, non-significant changes in the colon IL-23/IL-22 (Figures 3D,E) axis, while ZS2058 promoted the IL-23/IL-22 (Figures 3D,E) and IL-23/IL-17 (Figures 3D,F) axes. In summary, when compared with LGG, ZS2058 more strongly promoted the IL-23/IL-22 and IL-23/IL-17 axes in the host intestines. These axes might act synergistically to enhance host defense and promote pathogen clearance.

Although the host implements various anti-infection measures, Salmonella develops survival strategies such as unique respiration (Winter et al., 2010) and self-destructive cooperation (Ackermann et al., 2008). Consequently, surviving pathogens in the gut lumen adhere to the mucosal surface and initiate invasion. Salmonella attaches to the mucosal surface by binding to the mannose residues of glycoproteins with assistance from type 1 fimbriae in a process required for colonization of the host intestines (Erbslöh, 2013). As shown in Figure 2, the significant increase in MUC2 in response to Salmonella might contribute to this adhesion process. Compared with LGG, ZS2058 more efficiently reduced MUC2 production (Figure 2) in the colon, which might reduce the number of binding sites for Salmonella, thus discouraging adhesion and colonization and reducing the risks of invasion.

Taken together, the results listed above suggest that compared to LGG, ZS2058 more effectively reduces the pathogenicity of Salmonella in the intestinal phase. Therefore, pathogen control in the host gastrointestinal tract is required to prevent the further development of systemic infection and sepsis. Following intestinal infection, exogenous pathogens trigger a series of local inflammatory responses. Inflammation is a double-edged sword: an appropriate reaction favors the host's defense against infection, whereas an excessive response may cause unnecessary tissue damage. In the gut, inflammation was reported to provide a respiratory electron acceptor for Salmonella that would give it an advantage over the host microbiota (Winter et al., 2010). As shown in Figure 4A, Salmonella infection significantly increased the production of TNF- $\alpha$ in the mouse colon. This strong promoter of inflammatory responses suggests a tendency of Salmonella to provoke inflammation. LGG was previously reported to down-regulate TNF- $\alpha$ production in vivo (Mirpuri et al., 2012), and our observations with LGG and ZS2058 in the present study were consistent with those earlier findings
(Figure 4A). Notably, Tedelind et al. (2007) reported that PA could inhibit LPS-induced TNF- $\alpha$ production. Therefore, the increased PA (Table 1) levels in the colon might enhance the ability of ZS2058 to suppress TNF- $\alpha$ production (Figure 4A).

IL-10 is an anti-inflammatory Th2 cytokine that primarily inhibits the production of inflammatory cytokines by innate cells. During infection, IL-10 is required to avoid excessive immune response and prevent development of colitis (Howes et al., 2014). The slight increase in IL-10 (Figure 4B) production in the colons of infected mice might indicate a strategy by which the host controls local inflammatory responses. Notably, IL10 production in the colons of infected mice was suppressed by ZS2058 and maintained by LGG (Figure 4B), suggesting that the latter probiotic might better control inflammation in the infected gut. We also evaluated the production of MPO, an indicator of neutrophil filtration and colitis severity, in the colons of infected mice. As shown in Figure 4C, Salmonella infection and pretreatment with ZS2058 or T had no significant effect on MPO production in the colon. Consistent with a previous report in which LGG reduced MPO production in the lungs (Li et al., 2009), this study revealed a reduction in colonic MPO in mice treated with LGG (Figure 4C). In a murine model, LGG exacerbated the development of DSS-induced colitis and caused animal death (Mileti et al., 2009). However, a clinical study of patients with ulcerative colitis suggests that LGG can effectively and safely maintain remission (Zocco et al., 2006). Based on this study, we suggest that LGG is better able to alleviate gut inflammation during Salmonella infection. On one hand, these effects reduced the growth and competitive advantages of Salmonella vs. the gut microbiota in an inflammatory environment. On the other hand, excessive tissue damage was averted by the restriction of inflammatory responses.

After crossing the intestinal barrier, salmonellae are phagocytized by host immune cells such as macrophages. The bacteria replicate within these cells and are transported to other internal organs and into the blood (Broz et al., 2012), leading some invasive bacteria to shift from the intestinal phase to a systemic phase of infection. At this stage of infection, the in vivo response involves Th1-inducing cytokines that protect against Salmonella infection (Mizuno et al., 2003). Of these cytokines, IFN- $\gamma$ is among the most powerful first-line defense agents against Salmonella. IFN- $\gamma$ limits the availability of iron, withdraws iron from intracellular Salmonella (Nairz et al., 2008) and is reported to promote the intracellular killing of bacteria by activating neutrophils and macrophages (Van De Veerdonk et al., 2011). A reduced level of IFN- $\gamma$ (Figure 5A) in response to infection might enhance the intracellular survival of Salmonella and contribute to the development of systemic infection. Pretreatment with ZS2058, T or LGG restored the production of IFN- $\gamma$ (Figure 5A), which suggests that these lactobacillus strains help to promote the clearance of intracellular bacteria. As mentioned, IL-18 and IL-12 both induce IFN- $\gamma$ production. Our analyses found that both ZS2058 and T restored IFN- $\gamma$ production through an IL-18-dependent pathway (Figure 5B), whereas LGG induced the same effect through an IL-12dependent pathway (Figure 5C). These results demonstrate 
that the similar effects of probiotics against pathogenic invasion can be mediated by different mechanisms.

Based on the different effects of ZS2058 and LGG on IL18 and IL-12 production, we hypothesized that these probiotics might modify host immune responses toward pathogens via distinct mechanisms. Our analysis of several cytokines involved in host defense against infection revealed distinct cytokine profiles (Figure 7) in mice pretreated with ZS2058, compared to those pretreated with LGG. Although we did not definitively determine the functions of these modifications in the development and prevention of salmonellosis, we were able to demonstrate the distinct immunoregulatory effects of ZS2058 and LGG to mediate their similar abilities to prevent Salmonella infection in vivo.

In this study, the previously reported preventive effects of ZS2058 and LGG against Salmonella were further verified by the observation of reduced CRP levels in infected mice. We additionally investigated and compared the mechanisms underlying the effects of these two probiotic strains. We found that ZS2058 significantly increased the fecal PA level, increased the colonic MUC2 level and promoted the IL-23/IL-22 and IL23/IL-17 axes. Our results suggest that compared to LGG, ZS2058 more efficiently reduces the pathogenicity of Salmonella. An analysis of the cytokines in the infected mouse colon revealed

\section{REFERENCES}

Ackermann, M., Stecher, B., Freed, N. E., Songhet, P., Hardt, W. D., and Doebeli, M. (2008). Self-destructive cooperation mediated by phenotypic noise. Nature 454, 987-990. doi: 10.1038/nature07067

Alshawabkeh, K., and Tabbaa, M. J. (2002). Using dietary propionic acid to limit Salmonella gallinarum colonization in broiler chicks. Asian-Australas. J. Anim. Sci. 15, 243-246. doi: 10.5713/ajas.2002.243

Berndt, B. E., Zhang, M., Owyang, S. Y., Cole, T. S., Wang, T. W., Luther, J., et al. (2012). Butyrate increases IL-23 production by stimulated dendritic cells. Am. J. Physiol. Gastrointest. Liver Physiol. 303, G1384-G1392. doi: 10.1152/ajpgi. 00540.2011

Broz, P., Ohlson, M. B., and Monack, D. M. (2012). Innate immune response to Salmonella typhimurium, a model enteric pathogen. Gut Microbes 3, 62-70. doi: 10.4161/gmic.19141

Castillo, N.A., De Leblanc, A.D., Galdeano, C.M., and Perdigon, G. (2012). Probiotics: an alternative strategy for combating salmonellosis immune mechanisms involved. Food Res. Int. 45, 831-841. doi: 10.1016/j.foodres.2011. 04.031

De Keersmaecker, S. C. J., Verhoeven, T. L. A., Desair, J., Marchal, K., Vanderleyden, J., and Nagy, I. (2006). Strong antimicrobial activity of Lactobacillus rhamnosus GG against Salmonella typhimurium is due to accumulation of lactic acid. Fems Microbiol. Lett. 259, 89-96. doi: 10.1111/j. 1574-6968.2006.00250.x

Eckmann, L., and Kagnoff, M. F. (2001). Cytokines in host defense against Salmonella. Microbes Infect 3, 1191-1200. doi: 10.1016/S1286-4579(01) 01479-4

Erbslöh, V. (2013). Methods and Technologies to Reduce or Prevent Salmonella Infection Using Feed Additives in Poultry and Swine Production. Gödöllõ: Szent István University.

Howes, A., Stimpson, P., Redford, P., Gabrysova, L., and O'garra, A. (2014). Interleukin-10: Cytokines in Anti-Inflammation and Tolerance. Springer, Berlin. doi: 10.1007/978-4-431-54442-5_13

Hudault, S., Lievin, V., Bernetcamard, M. F., and Servin, A. L. (1997). Antagonistic activity exerted in vitro and in vivo by Lactobacillus casei (strain GG) against Salmonella typhimurium C5 infection. Appl. Environ. Microbiol. 63, 513-518. that LGG more effectively alleviated gut inflammation. Both probiotics restored the production of IFN- $\gamma$, a strong promoter of intracellular bacteria clearance, albeit by distinct pathways. Our results therefore demonstrated that ZS2058 and LGG use different mechanisms to exhibit similar anti-Salmonella effects in a murine model.

\section{AUTHOR CONTRIBUTIONS}

JL designed the study, performed research, analyzed data, and prepared the manuscript. ZG supervised the study and revised the manuscript. FS performed research and interpreted results. $\mathrm{HZ}$, JZ, and WC contributed to scientific idea and procedure designing of the study.

\section{FUNDING}

This work was supported by the National Natural Science Foundation of China (Nos. 31470161 and 31530056, WC), the national first-class discipline program of Food Science and Technology (JUFSTR20180102) and collaborative innovation center of food safety and quality control in Jiangsu Province.

Kemgang, T. S., Kapila, S., Shanmugam, V. P., Reddi, S., and Kapila, R. (2016). Fermented milk with probiotic Lactobacillus rhamnosus S1K3 (MTCC5957) protects mice from salmonella by enhancing immune and nonimmune protection mechanisms at intestinal mucosal level. J. Nutri. Biochem. 30, 62-73. doi: 10.1016/j.jnutbio.2015.11.018

Kumar, M., Kissoon-Singh, V., Coria, A. L., Moreau, F., and Chadee, K. (2017). Probiotic mixture VSL\#3 reduces colonic inflammation and improves intestinal barrier function in Muc2 mucin-deficient mice. Am. J. Physiol. Gastrointest. Liver Physiol. 312, G34-G45. doi: 10.1152/ajpgi.00298.2016

Lawhon, S. D., Maurer, R., Suyemoto, M., and Altier, C. (2002). Intestinal shortchain fatty acids alter Salmonella typhimurium invasion gene expression and virulence through BarA/SirA. Mol. Microbiol. 46, 1451-1464. doi: 10.1046/j. 1365-2958.2002.03268.x

Levison, M. E. (1973). Effect of colon flora and short-chain fatty acids on growth in vitro of Pseudomonas aeruginsoa and Enterobacteriaceae. Infect. Immun. 8, 30-35.

Li, N., Russell, W. M., Douglas-Escobar, M., Hauser, N., Lopez, M., and Neu, J. (2009). Live and heat-killed Lactobacillus rhamnosus GG: effects on proinflammatory and anti-inflammatory cytokines/chemokines in gastrostomy-fed infant rats. Pediatr. Res. 66, 203-207. doi: 10.1203/PDR. ob013e3181aabd4f

Li, X., Xu, Q., Jiang, T., Fang, S., Wang, G., Zhao, J., et al. (2016). A comparative study of the antidiabetic effects exerted by live and dead multi-strain probiotics in the type 2 diabetes model of mice. Food Funct. 7, 4851-4860. doi: 10.1039/ c6fo01147k

Liu, J., Hu, D., Chen, Y., Huang, H., Zhang, H., Zhao, J., et al. (2018). Strain-specific properties of Lactobacillus plantarum for prevention of Salmonella infection. Food Funct. 9 3673-3682. doi: 10.1039/c8fo00365c

McDade, T. W., Rutherford, J. N., Adair, L., and Kuzawa, C. (2008). Adiposity and pathogen exposure predict C-reactive protein in Filipino women. J. Nutr. 138, 2442-2447. doi: 10.3945/jn.108.092700

Mileti, E., Matteoli, G., Iliev, I. D., and Rescigno, M. (2009). Comparison of the immunomodulatory properties of three probiotic strains of Lactobacilli using complex culture systems: prediction for in vivo efficacy. PLoS One 4:e7056. doi: 10.1371/journal.pone.0007056

Mirpuri, J., Sotnikov, I., Myers, L., Denning, T. L., Yarovinsky, F., Parkos, C. A., et al. (2012). Lactobacillus rhamnosus (LGG) regulates IL-10 signaling in 
the developing murine colon through upregulation of the IL-10R2 receptor subunit. PLoS One 7:e51955. doi: 10.1371/journal.pone.0051955

Mizuno, Y., Takada, H., Nomura, A., Jin, C. H., Hattori, H., Ihara, K., et al. (2003). Th1 and Th1-inducing cytokines in Salmonella infection. Clin. Exp. Immunol. 131, 111-117. doi: 10.1046/j.1365-2249.2003.02060.x

Nairz, M., Fritsche, G., Brunner, P., Talasz, H., Hantke, K., and Weiss, G. (2008). Interferon-gamma limits the availability of iron for intramacrophage Salmonella typhimurium. Eur. J. Immunol. 38, 1923-1936. doi: 10.1002/eji. 200738056

Neill, M. A., Opal, S. M., Heelan, J., Giusti, R., Cassidy, J. E., White, R., et al. (1991). Failure of ciprofloxacin to eradicate convalescent fecal excretion after acute salmonellosis: experience during an outbreak in health care workers. Ann. Intern. Med. 114, 195-199. doi: 10.7326/0003-4819-114-3- 195

Pascual, M., Hugas, M., Badiola, J. I., Monfort, J. M., and Garriga, M. (1999). Lactobacillus salivarius CTC2197 prevents Salmonella enteritidis colonization in chickens. Appl. Environ. Microbiol. 65, 4981-4986.

Raffatellu, M., George, M. D., Akiyama, Y., Hornsby, M. J., Nuccio, S. P., Paixao, T. A., et al. (2009). Lipocalin-2 resistance confers an advantage to Salmonella enterica serotype Typhimurium for growth and survival in the inflamed intestine. Cell Host. Microbe 5, 476-486. doi: 10.1016/j.chom.2009.03.011

Rajashekara, G., Haverly, E., Halvorson, D. A., Ferris, K. E., Lauer, D. C., and Nagaraja, K. V. (2000). Multidrug-resistant Salmonella Typhimurium DT104 in poultry. J. Food Protect. 63, 155-161. doi: 10.4315/0362-028x-63.2.155

Ridker, P. M. (2003). Clinical application of C-reactive protein for cardiovascular disease detection and prevention. Circulation 107, 363-369. doi: 10.1161/01. CIR.0000053730.47739.3C

Santos, R. L., Raffatellu, M., Bevins, C. L., Adams, L. G., Tukel, C., Tsolis, R. M., et al. (2009). Life in the inflamed intestine, Salmonella style. Trends Microbiol. 17, 498-506. doi: 10.1016/j.tim.2009.08.008

Sunkara, L. T., Achanta, M., Schreiber, N. B., Bommineni, Y. R., Dai, G., Jiang, W., et al. (2011). Butyrate enhances disease resistance of chickens by inducing antimicrobial host defense peptide gene expression. PLoS One 6:e27225. doi: 10.1371/journal.pone.0027225

Tanner, S. A., Chassard, C., Rigozzi, E., Lacroix, C., and Stevens, M. J. A. (2016). Bifidobacterium thermophilum RBL67 impacts on growth and virulence gene expression of Salmonella enterica subsp enterica serovar Typhimurium. BMC Microbiology 16.46 doi: 10.1186/s12866-016-0659-x
Tedelind, S., Westberg, F., Kjerrulf, M., and Vidal, A. (2007). Anti-inflammatory properties of the short-chain fatty acids acetate and propionate: a study with relevance to inflammatory bowel disease. World J. Gastroenterol. 13, 2826-2832. doi: $10.3748 /$ wjg.v13.i20.2826

Valeri, M., and Raffatellu, M. (2016). Cytokines IL-17 and IL-22 in the host response to infection. Pathog. Dis. 74:ftw111. doi:10.1093/femspd/ $\mathrm{ftw111}$

Van De Veerdonk, F. L., Netea, M. G., Dinarello, C. A., and Joosten, L. A. (2011). Inflammasome activation and IL-1beta and IL-18 processing during infection. Trends Immunol. 32, 110-116. doi: 10.1016/j.it.2011.01.003

Winter, S. E., Thiennimitr, P., Winter, M. G., Butler, B. P., Huseby, D. L., Crawford, R. W., et al. (2010). Gut inflammation provides a respiratory electron acceptor for Salmonella. Nature 467, 426-429. doi: 10.1038/nature 09415

Yeung, C.Y., Chiau, J.S.C., Chan, W.T., Jiang, C.B., Cheng, M.L., Liu, H.L., et al. (2013). In vitro prevention of Salmonella lipopolysaccharide-induced damages in epithelial barrier function by various lactobacillus strains. Gastroenterol. Res. Pract. 3:973209. doi: 10.1155/2013/973209

Yoshimi, N., Matsunaga, K., Katayama, M., Yamada, Y., Kuno, T., Qiao, Z., et al. (2001). The inhibitory effects of mangiferin, a naturally occurring glucosylxanthone, in bowel carcinogenesis of male F344 rats. Cancer Lett. 163, 163-170. doi: 10.1016/S0304-3835(00)00678-9

Zocco, M. A., Dal Verme, L. Z., Cremonini, F., Piscaglia, A. C., Nista, E. C., Candelli, M., et al. (2006). Efficacy of lactobacillus GG in maintaining remission of ulcerative colitis. Aliment Pharmacol. Ther. 23, 1567-1574. doi: 10.1111/j. 1365-2036.2006.02927.x.

Conflict of Interest Statement: The authors declare that the research was conducted in the absence of any commercial or financial relationships that could be construed as a potential conflict of interest.

Copyright ( 2019 Liu, Gu, Song, Zhang, Zhao and Chen. This is an open-access article distributed under the terms of the Creative Commons Attribution License (CC BY). The use, distribution or reproduction in other forums is permitted, provided the original author(s) and the copyright owner(s) are credited and that the original publication in this journal is cited, in accordance with accepted academic practice. No use, distribution or reproduction is permitted which does not comply with these terms. 